\title{
RLASCA - A Novel technique for cerebral blood flow imaging
}

\author{
D. D. H. Meer Khalander ${ }^{1}$, A. Sathish ${ }^{2}$ \\ ${ }^{I}$ (M.Tech[DSCE], RGMCET, Nandyal, Andhra Pradesh \\ ${ }_{2}^{2}$ (A. Sathish, Asso.Professor, RGMCET, Nandyal, Andhra Pradesh)
}

\begin{abstract}
Detection of cerebral blood flow $(C B F)$ is vital and Conventional technique that is used for in vivo detection of CBF is Laser Speckle Imaging (LSI). LSI is a two dimensional noninvasive optical imaging technique that is widely used to study rat's cerebral blood flow (CBF) under a variety of physiological and pathological conditions. LSI does not consider the influence of these minute disturbances caused due to respiration and/or heart beat. In this paper we analyze how these physiologic motions affect the spatial resolution of conventional Laser speckle contrast analysis (LASCA). We propose a Novel registration technique called registered laser speckle contrast analysis (RLASCA) method which first registers the raw speckle image through $3 \times 3$ convolution kernel, normalize correlation metric and B-spline interpolator and constructs the contrast image for cerebral blood flow. This RLASCA improves the not only improves the distinguishability of small vessels in the image and suppresses the noise caused by respiration and/or heart beating. In an experiment of imaging the angiogenesis of rat's brain tumor, all data were analyzed by both traditional LASCA and RLASCA; RLASCA outperformed the conventional LASCA in providing a much higher resolution for new small vessels in cerebra. As a processing method for LSI, RLASCA can be directly applied to other LSI experiments where the disturbances from different sources (like respiration, heart beating) exist. The proposed work is to be developed on Matlab platform.
\end{abstract}

Keywords: Cubic B-spline interpolation, laser speckle imaging (LSI), normalized correlation registration, registered laser speckle contrast analysis (RLASCA).

\section{INTRODUCTION}

As a 2-D and noninvasive optical imaging technique, laser speckle imaging (LSI) has been widely used to study rat's cerebral blood flow (CBF) under a variety of physiological and pathological conditions, e.g., focal cerebral ischemia, hypotension, peripheral electrical stimulation, hypothermia and brain tumor. When the tissue contains scattering moving particles, e.g., blood cells, sequentially recorded raw speckle images $\left\{I_{i}(x, y)\right\}(i=$ $1, \ldots, N$ are analyzed by laser speckle contrast analysis (LASCA) to obtain both CBF and vessel structure information. According to the theory of LASCA, the contrast value $K^{2}$, defined as the square of ratio of standard deviation $\sigma$ to mean intensity $\langle I\rangle$, is inversely proportional to velocity $v$ (1)

$$
K^{2}=\left(\frac{\sigma}{\langle I\rangle}\right)^{2} \propto \frac{1}{v}
$$

$K^{2}$ can be estimated in either spatial $K_{s}^{2}$ or temporal $K_{t}^{2}$ formalism. For each frame of the acquired speckle images, $K_{s}^{2}$ for each pixel is calculated based on the intensities in a small window (typically $7 \times 7$ ), and therefore loses the spatial resolution. Similarly, $K_{t}^{2}$ at each pixel is estimated using the intensities of the pixel in continuously recorded raw speckle images. In practice, 50 or more frames are used for statistical efficiency. As a result, $K_{t}^{2}$ retains the spatial resolution but temporal resolution is compromised.

Like other optical imaging techniques, LSI is also susceptible to kinds of mechanical vibrations within the workspace. Such mechanical vibrations can be minimized by using vibration isolated optical platform and other precautions. In rodent in vivo experiments, the animals are always anesthetized and constrained in a stereotaxic frame to suppress movements. However, there are still some inevitable disturbances in raw speckle images due to respiration and/or heart beating of the animals. Such global disturbances could lead to the following problems in LASCA: 1) the loss of spatial resolution, the output of LASCA is going to be blurred, and thus it is more difficult to distinguish small vessels from tissue; 2) inaccurate estimation of contrast values, disturbances change the statistic property of the speckles and lead to inaccurate estimation of CBF by LASCA. In order to overcome these problems, we propose to register the raw speckle images before LASCA, or called registered LASCA (RLASCA) hereafter. Since the raw speckle images are usually too noisy to find a stable pattern for registration, we develop a novel automatic registration technique, using a $3 \times 3$ convolution kernel, a normalized correlation metric and a cubic B-spline interpolator, to register raw speckle images accurately. RLASCA is shown to enhance the small vessels and alleviate noises in both tissue area and vessels. As an 
application, we studied the angiogenesis in rat's brain tumor by LSI, and showed how RLASCA improves the resolution of cortical vascular structure of the angiogenesis.

\section{METHOD}

RLASCA involves two steps: 1) registering raw speckle images $\left\{I_{i}(x, y)\right\}(i=1, \ldots, N)$ and 2$)$ calculating LASCA based on the registered raw speckle images $\left\{\hat{I}_{i}(x, y)\right\}(i=1, \ldots, N)$.

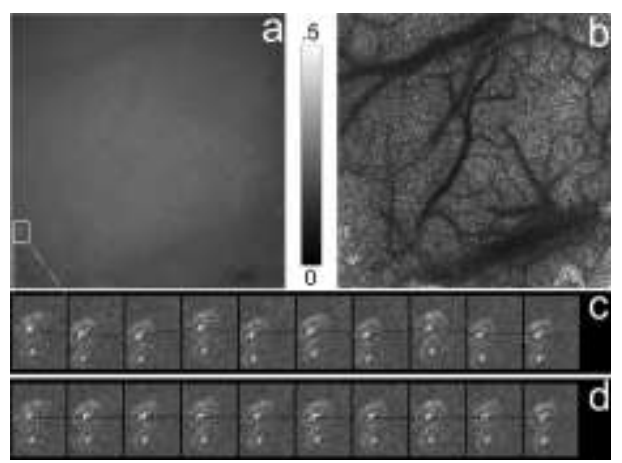

Fig. 1. (a) One typical raw speckle image. (b) Preprocessed image of (a) using a $3 \times 3$ convolution kernel. (c) Zoomed out images of white box area in first ten frames with obvious disturbances. (d) Images of the same area after registration with eliminated disturbances.

\section{A. Registering Raw Speckle Images by Modified Registration Technique}

Traditional registration techniques are incapable of processing raw speckle images $\left\{I_{i}(x, y)\right\}(i=$ $1, \ldots, N)$, because the random speckles indicate no stable pattern, which could be used for registration [see Fig. 1(a)]. Occasionally, there are some isolated pixels overexposed due to strong reflection [e.g., white boxed area in Fig. 1(a)], in which case, the continuously acquired speckle images can be registered based on these overexposed pixels. However, in practice, the existence of such overexposed area is neither guaranteed nor desired.

In this paper, we propose a novel registration technique to register the raw speckle images. First, each raw speckle image $\left\{I_{i}(x, y)\right\}(i=1, \ldots, N)$ is preprocessed by a $3 \times 3$ convolution kernel as described in the following to reveal vessel structure pattern. Then a rigid translation parameter $\left(\Delta x_{i}, \Delta y_{i}\right)$ is estimated for each preprocessed image $S_{i}(x, y)(i=1, \ldots, N)$ by a normalized correlation metric. Finally, the raw speckle images $\left\{I_{i}(x, y)\right\}(i=1, \ldots, N)$ are registered (re sampled) by a cubic B-spline interpolator based on their rigid translation parameters $\left(\Delta x_{i}, \Delta y_{i}\right)$ to obtain the registered raw speckle images $\left\{I_{i}(x, y)\right\}(i=1, \ldots, N)$.

1) Preprocessing with $3 \times 3$ Convolution Kernel: A $3 \times 3$ convolution kernel (2) is implemented to preprocess each raw speckle image to obtain the spatial standard deviation at each pixel, which is related to vessel structure information (3)

$$
\begin{gathered}
\text { Kernel }=\frac{1}{9}\left(\begin{array}{lll}
1 & 1 & 1 \\
1 & 1 & 1 \\
1 & 1 & 1
\end{array}\right) \\
S_{i}^{2}=\left(I_{i}^{2} \otimes \text { Kernel }\right)-\left(I_{i} \otimes \text { Kernel }\right)^{2} .
\end{gathered}
$$

Here for a given matrix $A$ with pixel index $(x, y), A^{2}$ represents $A^{2}(x, y)=(A(x, y))^{2}$.

Based on (3), the size of image $S_{i}$ is equal to that of $I_{i}$. Since the convolution kernel size is $3 \times 3$, only the information at the four borders (the first row, the first column, the last row, and the last column) of $S_{i}$ is lost. Therefore, the average loss of spatial resolution in this preprocessing step is very small and always less than one pixel. The preprocessed images [see Fig. 1(b)], i.e., $S_{i}(x, y)(i=1, \ldots, N)$, reveal the same vessel structure pattern which, therefore, can be used for registering $\left\{I_{i}(x, y)\right\}(i=1, \ldots, N)$ with a minor loss of spatial resolution.

2) Obtaining Rigid Translation Parameters Based on Normalized Correlation Metric: In this step, each image $S_{i}(i=2, \ldots, N)$ is to be registered to the first image $S_{i}$ in accordance with a proper rigid translation. The optimized rigid translation parameter $\left(\Delta x_{i}, \Delta y_{i}\right)$ for each $S_{i}(i=2, \ldots, N)$ is determined by maximizing the 
normalized correlation metric (4) using a gradient descent optimization procedure [10] with a variable step length

$$
\text { Metric }=\frac{\sum_{x, y} S_{1}(x, y) S_{i}\left(x-\Delta x_{i}, y-\Delta y_{i}\right)}{\sqrt{\sum_{x, y} S_{1}^{2}(x, y) \sum_{x, y} S_{i}^{2}\left(x-\Delta x_{i}, y-\Delta y_{i}\right)}}
$$

Among various registration metrics, we have chosen the normalized correlation metric for its advantages of low computational cost and well-defined maxima (sharp peaks) in the cost function. All rigid translation parameters $\left(\Delta x_{i}, \Delta y_{i}\right)$ are used to register (resample) the corresponding raw speckle images $\left\{I_{i}(x, y)\right\}(i=1, \ldots, N)$ in the next step.

3) nResampling Raw Speckle Images Using Cubic B-Spline Interpolator: The displacement due to disturbances need not conform to integral pixels, so the rigid translation parameters $\left(\Delta x_{i}, \Delta y_{i}\right)$ always have sub pixel precision. Therefore, a resampling interpolation is needed to register the raw speckle images $\left\{I_{i}(x, y)\right\}(i=1, \ldots, N)$. In this study, the cubic B-spline interpolator is used to calculate the intensity of non grid position in a two dimensional piecewise polynomial way based on the cubic B-spline function (5) which is smooth, continuous and bounded

$$
\beta^{3}(d)= \begin{cases}2 / 3-|d|^{2}(2-|d|) / 2, & 0 \leq|d|<1 \\ \left(2-|d|^{3}\right) / 6, & 0 \leq|d|<1 \\ 0, & 0 \leq|d|<1\end{cases}
$$

where $|d|$ is the distance from the interpolated point.

Although the computational cost of B-spline interpolation is not very low, it is used in this study because cubic $\mathrm{B}$-spline interpolation utilizes the non-integer parameter which is preferable over the nearest neighbor interpolation. In addition, cubic B-spline interpolation prevents the effect of degeneration in linear interpolation. After resampling, the registered raw speckle images $\hat{I}_{i}(i=1, \ldots, N)$ are obtained. The above registration procedures are summarized in Fig. 2.

\section{B. Temporal LASCA Based on Registered Speckle Images}

After registration as described earlier, the registered raw speckle images $\hat{I}_{i}(i=1, \ldots, N)$ are then analyzed by conventional temporal LASCA to obtain maps of blood vessels and flow. The whole procedure as described earlier is then called registered LASCA (RLASCA).

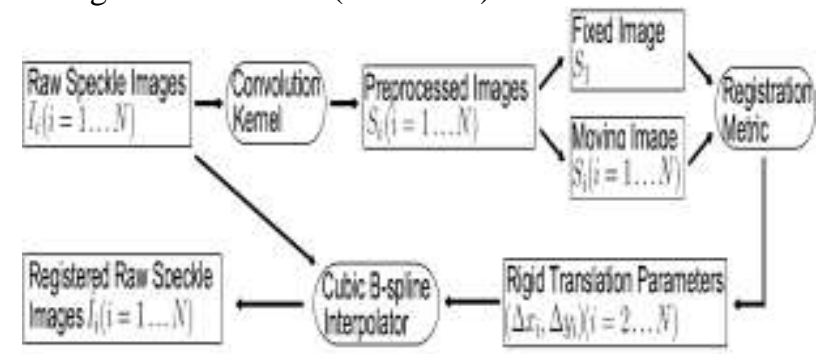

Fig.2. Registration procedure based on the modified registration method.

\section{A. Animal Preparation}

\section{Experiment And Data Analysis}

The experimental protocol used in this study has been approved by the Animal Care and Use Committee of Johns Hopkins Medical Institutions. The female Sprague-Dawley (SD) rat ( $325 \mathrm{~g})$ was anesthetized with intraperitoneal (IP) injection of a mixture of $90 \mathrm{mg} / \mathrm{kg}$ of ketamine and $10 \mathrm{mg} / \mathrm{kg}$ of xylazine. The rat was constrained in a stereotaxic frame (model 975, Kopf Instruments, Tujunga, CA). A midline incision was made over the scalp and the tissues over the bones were cleaned with a blade. A $6 \times 6 \mathrm{~mm} 2$ cranial window overlying the right somatosensory cortex (centered at $4.5 \mathrm{~mm}$ lateral and $2.5 \mathrm{~mm}$ posterior to the bregma) was thinned with a high speed dental drill (Fine Science Tools, Inc., North Vancouver, Canada) with $1.4 \mathrm{~mm}$ steel burr until half transparent. Saline was used to cool down the scalp during the surgery. Rectal temperature was maintained at 37 .C throughout the experiment using a Gaymar Heat Therapy System (model TP-500 T/Pump, Gaymar Industries, Inc., New York, NY). 
In the brain tumor angiogenesis experiment, the female Fisher 344 rats $(\sim 170 \mathrm{~g})$ were similarly prepared for imaging. In addition, a $6 \times 6 \mathrm{~mm} 2$ cranial window was thinned on the contra lateral left cortex (centered at $3.5 \mathrm{~mm}$ lateral and $3 \mathrm{~mm}$ posterior to the bregma). After recording the baseline raw speckle images on day 0 , a hole was drilled carefully at the center of the cranial window with a $1 \mathrm{~mm}$ steel burr until the dura was reached. Then 100000 cells of $9 \mathrm{~L}$ glioma were injected into the cortex using a 26 gauge

Hamilton syringe at a depth of 2 mmbelow the surface of the cerebral cortex to induce brain tumor. The skin was closed with surgical clips and the rat was housed in the animal facility, as the tumor was allowed to grow.

A 12-bit, cooled CCD camera (Sensicam SVGA, Cooke, MI) with a $60 \mathrm{~mm}$ macro (1:1 maximum reproduction ratio) $\mathrm{f} / 2.8$ lens was focused on the blood vessels in the cranial window. Exposure time of the CCD was set to 5 $\mathrm{ms}$. Frame rate was $11 \mathrm{ft} / \mathrm{s}$. The imaging field was illuminated with a $632 \mathrm{~nm} \mathrm{He}-\mathrm{Ne}$ Laser beam source (1508P$\mathrm{O}$, Uniphase, $\mathrm{CA}$ ). The laser beam was reshaped by a lens to illuminate the entire thinned skull window. The field of view recorded by the CCD camera was $704 \times 704$ pixels corresponding to an imaging area of $4.7 \times 4.7$ $\mathrm{mm} 2$ on the rat's brain.

\section{B. Data Recording and Processing}

In the SD rat experiment, a white light reflectance image (40- ms exposure time) was obtained first for reference. Following that, a stack of 80 raw speckle images were acquired sequentially. In the brain tumor experiment, the baseline recordings were performed on the day of tumor inoculation (day 0). After that,

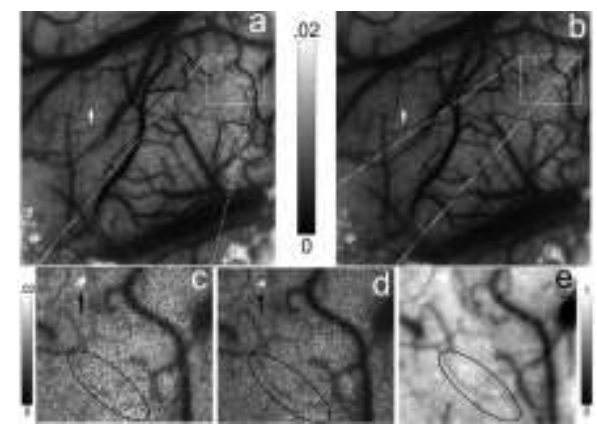

Fig. 3. RLASCA improves the spatial resolution of contrast image. (a) Contrast image based on original unregistered raw speckle images. (b) Contrast image of RLASCA. The white arrows in (a) and (b) indicate the same small vessel which can be seen more clearly in (b) than in (a). (c) and (d) Zoomed out images of the white box area in (a) and (b), respectively. The black arrows in (c) and (d) show the suppression of blur effect. (e) White light image of the corresponding area.

on day 10, the Fisher rat was imaged again to investigate the angiogenesis. All data were analyzed by both traditional LASCA and RLASCA.

\section{RESULTS}

Fig. 1(a) shows an example of the raw speckle images acquired by the camera. Fig. 1(c) zooms out the white box area in Fig. 1(a) with some overexposed pixels in the first ten frames. The disturbances among the images are obvious. After the registration [see Fig. 1(d)], the effect of such disturbances is removed.

\section{A. Revealing Small Vessels Under Higher Resolution}

Fig. 3(a) and (b) shows the contrast images based on unregistered $\left(K^{2}\right)$ and registered $\left(\widehat{K}^{2}\right)$ raw speckle images, respectively. Although the major vascular structures in $K^{2}$ are similar to that in $\widehat{K}^{2}$, smaller vessels can be recognized more clearly in $\widehat{K}^{2}$ [white arrows in Fig. 3 (a) and (b)] after eliminating the disturbances. The over-exposed area (black arrows) in Fig. 3(d) processed by RLASCA is smaller than that in Fig. 3(c) processed by LASCA, implying that the blur effect is eliminated. Human vision system produces the most efficient segmentation output [14] which is always used as the gold standard for image segmentations. To obtain the gold standard, ten human observers (all of them are graduate students at johns Hopkins university with fair understanding of

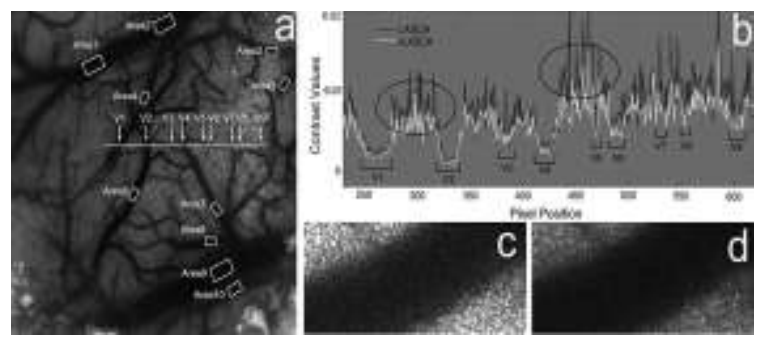


Fig.4. RLASCA alleviates the noise derived from respiration and heart beating. (a) Contrast image by RLASCA. Ten areas (Area 1-Area 10 ) in the center portion of different vessels are selected for quantitative analysis. (b) Contrast values along the white line in (a) based on unregistered (black curve) and registered (white curve) raw speckle images. The white line in (a) passes nine vessels (V1- V9) identified by a human observer. Two black circles show that the noise is suppressed by RLASCA. (c) and (d) Zoomed out images of Area 1 in the contrast images based on unregistered and registered raw speckle images, respectively.

vascular images) were randomly divided into two groups. Fig. 3(c) was presented to the observers in the first group, while observers in the second group were asked to look at Fig. 3(d). All observers needed to judge if there is a vessel in the black circled area. Only one observer in the first group thought there was a vessel within the circle in Fig. 3(c), while the second group unanimously observed a vessel within the same area in Fig. 3(d). This experiment indicated that the small vessel could only be distinguished by RLASCA. Fig. 3(e) shows the white light image of the corresponding area as the ground truth image.

\section{B. Alleviating Noises in Both Tissue and Vessel Areas}

The disturbances may result in unreliable contrast values, which could exist in both the tissue and vessel areas.

Fig. 4(b) shows the contrast values along the white line in Fig. 4(a). The contrast values in $K^{2}$ and $\widehat{K}^{2}$ are shown in black and white colors, respectively.

The contrast values are inversely proportional to blood velocity, leading tissue areas always have higher contrast values (smaller velocity) than vessels (arterioles and venules). RLASCA alleviates the noise in the tissue area [e.g., circled area in Fig. 4(b)], while still preserving the distinguish ability between tissue and vessel.

To demonstrate the de-noising effect of RLASCA, we select nine blood vessels (labeled V1-V9) crossing the white line in Fig. 4(a). The disturbances could result in wrong flow pattern in the vessel areas. As shown in Fig. 4(b), $\widehat{K}^{2}$ by RLASCA clearly inhibited the noisy fluctuations in all nine vessel areas. In particular, in vessels V3 and V9, the noise is evidently reduced; the contrast values in vessels V6 and V8 are significantly smaller than that in the surrounding tissue area.

To quantitatively analyze the denoising performance of RLASCA, we manually selected ten areas (Area 1-Area 10) overlying the center portion of different vessels. Fig. 4(c) and (d) show the zoomed out image of Area1 in $K^{2}$ and $\widehat{K}^{2}$, respectively. Obviously, the conventional $K_{2}$ contains more noisy pixels in the center portion of the vessels as compared to $\widehat{K}^{2}$. To compare the level of noisy fluctuations in $K^{2}$ and $\widehat{K}^{2}$, we use the standard deviation (normalized by 10-4) of the contrast values in each selected area to measure the level of fluctuation due to noise. Larger standard deviation indicates higher fluctuation. The results are presented in Table I. It was found that the fluctuation level of $K^{2}$ was always higher than that of $\widehat{K}^{2}$. According to the right tail paired $t$-test, the fluctuation level of $K^{2}$ was significantly higher than that of $\widehat{K}^{2}(p<0$. 001), which indicating that RLASCA has a better denoising performance in vessel areas.

\section{Application in Investigating Brain Tumor Angiogenesis}

One of the advantages of RLASCA is to improve the spatial resolution of contrast images of cortical microvasculature. Such a high resolution view is very useful in assessing the changes of cortical vascular. In this study, we applied RLASCA to investigate the angiogenesis of rat's brain tumor. Fig. 5 shows the contrast images obtained using both LASCA [see Fig. 5(b)] and RLASCA [see Fig. 5(c)] on day 10. Compared with the baseline contrast image [see Fig. 5(a)] on day 0, there exists obvious angiogenesis of tumor on day 10. Fig. 5(d) and (e) shows the zoomed out images corresponding to the white boxes areas in Fig. 5(b) and (c), respectively. Using RLASCA, micro vessels resulting from angiogenesis can be distinguished more clearly from the tissue area [e.g., the white arrows in Fig. 5(e)]. In particular,

in some areas [e.g., the white circles in Fig. 5(d) and (e)], the small vessel and its branches can only be seen by RLASCA.

\section{A. Sources of Disturbances in LSI}

\section{Discussion}

To confirm that the disturbances do originate from respiration and heart beating, we ran similar experiments on hairless mice (strain SKH1). After anesthetizing the mouse, its right ear was rigidly taped onto a glass slide so that there was no effects from respiration and heart beating. Then, the mouse's ear was imaged using the same imaging procedure. Similar to the above procedure, 80 speckle images were recorded and analyzed by both LASCA [see Fig. 6(a)] and RLASCA [see Fig. 6(b)]. All the rigid translation parameters for registration are found to be much closed to zero, indicating no disturbances at all. Comparing $K_{2}$ with $^{\wedge} K$, there were no observable differences between Fig. 6(a) and (b), which further confirms that the main sources of the image disturbances are respiration and heart beating. This result indicates LASCA and RLASCA produce the same contrast image in the absence of disturbances. However, the source of disturbances is unavoidable in most in vivo imaging experiments, and thus need to be suppressed by RLASCA. It should be noted that the periodic 
noise can also be removed by filtering the images acquired at much higher sampling rate. However, in this study, the disturbances were mainly from respiration and heart beating after a thinned skull preparation. The frequency of noise originating from disturbances is difficult to be estimated in the temporal domain, so the filtering technique is not appropriate in this case. In practice, the disturbances may originate from other sources depending on the experiment conditions. For example, when imaging the rat's CBF with an

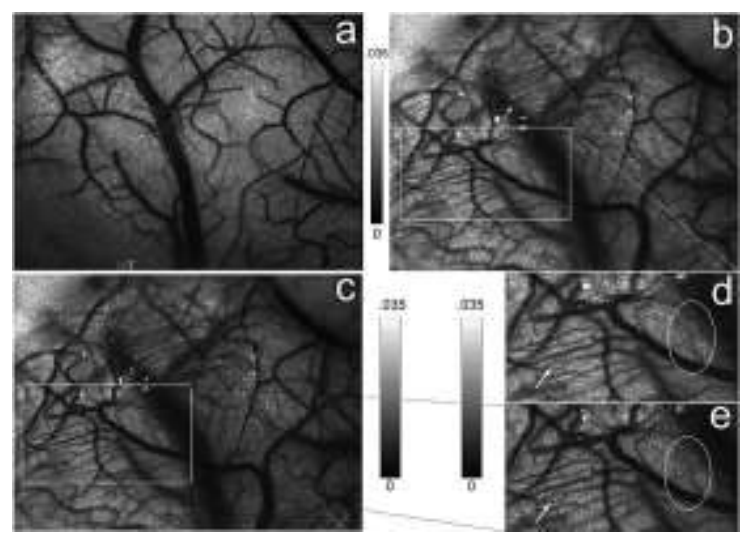

Fig.5. Application for investigating angiogenesis of rat's brain tumor: (a) RLASCA result based on the baseline raw speckle images recorded on day 0. (b) Contrast image based on the unregistered raw speckle images recorded on day 10. (c) RLASCA result based on the raw speckle images recorded on day 10. (d) and (e) Zoomed out images of the white box areas in (a) and (b), respectively.

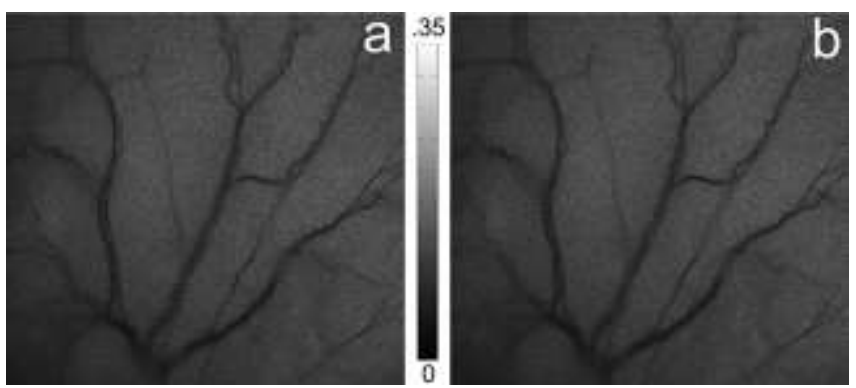

Fig.6. Laser speckle imaging of mouse's ear: (a) Contrast image based on the unregistered raw speckle images. (b) Contrast image by RLASCA.

Opened skull preparation, the changing of intracranial pressure may result in greater disturbances; similarly, if the experiment involves electrical stimulation, stimulation artifacts could be another source of disturbances. As a general tool, RLASCA can be directly applied to deal with different disturbances.

\section{B. Influences of Different Interpolators}

Interpolation is the key step in the registration of raw speckle images. Fig. 7 shows the contrast values along the white line in Fig. 4(a) based on the registered speckle images using different interpolators: 1) nearest neighbor interpolator (shown in black); 2) linear interpolator (shown in gray); and 3) cubic Bspline interpolator (shown in white). Registration with the nearest neighbor interpolator is actually based on the integer part of rigid translation parameters, therefore, disturbances can not be

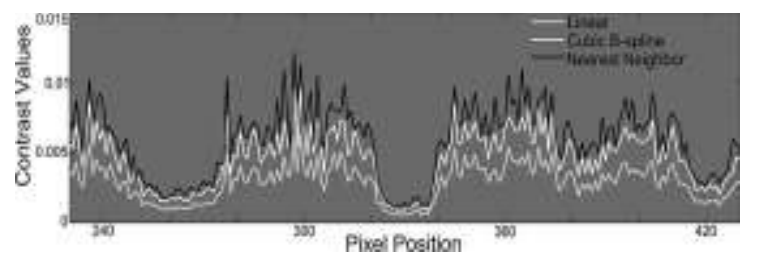

Fig. 7. Results of RLASCA along the white line in Fig. 4(a) using different interpolators: nearest neighbor interpolation (black curve), cubic B-spline interpolation (white curve), and linear interpolation (gray curve).

Eliminated efficiently. For linear interpolator, although there is no loss in the rigid translation parameters, the range of contrast values is compressed so that the distinguish ability between vessel and tissue is poor. The result of linear interpolation resembles a degenerated case of the result of cubic B-spline interpolation. Compared with the other two interpolators, cubic B-spline interpolator produces more accurate registration while preventing the degeneration of the distinguish ability between vessel and tissue. 


\section{CONCLUSION}

In this paper, we propose RLASCA to eliminate the effect of disturbances due to respiration and heart beating during imaging the rat's $\mathrm{CBF}$. We implemented a modified registration technique containing a $3 \times 3$ convolution kernel, normalized correlation metric and cubic B-spline interpolator to register the raw speckle images accurately. RLASCA improves the spatial resolution of contrast image and alleviates the noisy effect of disturbances. In the application for investigating angiogenesis of rat's brain tumor, RLASCA shows the small vessels with a higher resolution.

\section{References}

[1]. J. Briers, "Laser Doppler, speckle and related techniques for blood perfusion mapping and imaging," Physiol. Meas., vol. 22, no. 4, pp. $35-66$,

[2]. 2001

[3]. D. Zhu,W. Lu,Y.Weng, H. Cui, andQ. Luo, "Monitoring thermal-induced changes in tumor blood flow and microvessels with laser speckle contrast imaging," Appl. Opt., vol. 46, no. 10, pp. 1911-1917, 2007.

[4]. T.Durduran,M. Burnett, G.Yu, C. Zhou, D. Furuya,A.Yodh, J. Detre, and J. Greenberg, "Spatiotemporal quantification of cerebral blood flow during functional activation in rat somatosensory cortex using laser-speckle flowmetry," J. Cereb. Blood Flow Metab., vol. 24 , pp. $518-525,2004$.

[5]. A. Dunn, H. Bolay, M. Moskowitz, and D. Boas, "Dynamic imaging of cerebral blood flow using laser speckle," J. Cereb. Blood Flow Metab., vol. 21, pp. 195-201, 2001.

[6]. H. Cheng, Q. Luo, S. Zeng, S. Chen, J. Cen, and H. Gong, "Modified laser speckle imaging method with improved spatial resolution," J. Biomed. Opt., vol. 8, no. 3, pp. 559-564, 2003.

[7]. A. Kharlamov, B. Brown, K. Easley, and S. Jones, "Heterogeneous response of cerebral blood flow to hypotension demonstrated by laser speckle imaging flowmetry in rats," Neurosci. Lett., vol. 368, no. 2, pp. 151-156, 2004.

[8]. J. Parker, R. Kenyon, and D. Troxel, "Comparison of interpolating methods for image resampling," IEEE Trans. Med. Imag., vol. MI-2, no. 1,pp. 31-39, Mar. 1983.

[9]. M. Li, P. Miao, J. Yu, Y. Qiu, Y. Zhu, and S. Tong, "Influences of hypothermia on the cortical blood supply by laser speckle imagings,” IEEE Trans. Neural Syst. Rehabil. Eng., vol. 17, no. 2, pp. 128-134, Apr. 2009 